\title{
Attenuated SUMOylation of sirtuin 1 in premature neonates with bronchopulmonary dysplasia
}

\author{
FENGMEI TAN, WENBIN DONG, XIAOPING LEI, XINGLING LIU, \\ QINGPING LI, LAN KANG, SHUAI ZHAO and CHAN ZHANG
}

Department of Neonatology, Affiliated Hospital of Southwest Medical University, Luzhou, Sichuan 646000, P.R. China

Received March 6, 2017; Accepted September 9, 2017

DOI: $10.3892 / \mathrm{mmr} .2017 .8012$

\begin{abstract}
A prospective study was performed to investigate the effects of hyperoxia on the expression of small ubiquitin-related modifier (SUMO) and sirtuin 1 (SIRT1) proteins, and to examine interactions between these proteins in premature neonates with bronchopulmonary dysplasia (BPD). Peripheral blood mononuclear cells (PBMCs) were isolated from residual venous blood samples of 20 premature infants with BPD and 20 gender-matched premature infants without BPD (non-BPD group). Expression levels of SUMO and SIRT1 proteins in PBMCs were assessed by western blot analysis, and their interactions in PBMCs were detected using the immunoprecipitation assay. Based on the fraction of inspired oxygen $\left(\mathrm{FiO}_{2}\right)$ administered, neonates were divided into normoxia, low- $\left(21 \%<\mathrm{FiO}_{2}<30 \%\right)$, medium- $\left(30 \% \leq \mathrm{FiO}_{2}<40 \%\right)$ and high-oxygen $\left(\mathrm{FiO}_{2} \geq 40 \%\right)$ groups. Expression levels of SUMO1 and SUMO2/3 proteins in the normoxia group were significantly lower than those in the medium- or high-oxygen groups $(\mathrm{P}<0.01)$, but were comparable to those in the low-oxygen group. SIRT1 expression levels in both the medium- and high-oxygen groups were significantly lower than those in the normoxia group $(\mathrm{P}<0.01)$. In the BPD group, the expression of SIRT1 protein was significantly lower $(\mathrm{P}<0.01)$, and its interaction with SUMO1 and SUMO2/3 was significantly attenuated compared with that in the non-BPD group $(\mathrm{P}<0.01)$. Supplemental oxygen with $\mathrm{FiO}_{2} \geq 30 \%$ was associated with upregulation of SUMO1 and SUMO2/3 expression and downregulation of SIRT1 expression. The present findings suggest that decreased SIRT1 expression and its SUMOylation by SUMO1 and SUMO2/3 may be associated with the development of BPD.
\end{abstract}

Correspondence to: Dr Wenbin Dong, Department of Neonatology, Affiliated Hospital of Southwest Medical University, 25 Taiping Road, Luzhou, Sichuan 646000, P.R. China

E-mail: swmudwb@163.com

Key words: sirtuin 1, SUMOylation, bronchopulmonary dysplasia, premature neonates, oxygen inhalation

\section{Introduction}

Bronchopulmonary dysplasia (BPD) is a neonatal chronic lung disease characterized by impaired lung development $(1,2)$. The pathogenesis of BPD is complex, and is thought to be associated with a number of features, including genetic factors, premature birth, prenatal and postnatal infections, mechanical ventilation, and oxygen toxicity $(1,2)$. Supplemental oxygen is an important aspect of treatment of respiratory failure in premature neonates. However, oxidative stress induced by hyperoxic exposure results in overproduction of reactive oxygen metabolites (including hydrogen peroxide, singlet oxygen, superoxide free radicals, and hydroxyl free radicals), which overwhelms the immature antioxidant enzyme systems of premature neonates, causes lung injury, impairs alveolarization and leads to BPD $(3,4)$.

The mammalian sirtuin (SIRT) family is comprised of seven nicotinamide adenine dinucleotide (NAD)-dependent histone deacetylase (HDAC) members (5). Of these, SIRT1 is the most-studied protein and has a role in the regulation of several physiopathological processes, including gene expression, mitochondrial function, cellular metabolism and response to oxidative stress (6-10). Furthermore, SIRT1 is known to protect against oxidative stress-induced cell proliferation and inhibit cell apoptosis $(7,8,11-13)$.

Conjugation of small ubiquitin-related modifier (SUMO) to target proteins, which is also referred to as SUMOylation, is an important post-translational modification that regulates a variety of cellular processes (14). Similar to ubiquitylation, SUMOylation requires E1 (activating), E2 (conjugating) and E3 (ligating) enzymes to conjugate SUMO to its substrates (15). The mammalian genome encodes four SUMO isoforms (SUMO1, SUMO2, SUMO3, and SUMO4) $(16,17)$. Of these isoforms, SUMO2 and SUMO3 are similar in structure and are referred to as SUMO2/3; SUMO1 is $\sim 50 \%$ similar to SUMO2/3, whereas SUMO4 shares $87 \%$ sequence identity with SUMO2/3 $(16,17)$. SUMO is crucial for oxidative stress responses and SUMOylation is one of the biological processes triggered by oxidative stress (15). An increasing number of SUMOylated proteins have been identified in cells exposed to stress, including oxidative stress, heat shock, DNA damage and ethanol stress $(14,18,19)$.

In a previous study, lower expression of SIRT1 in leukocytes isolated from tracheal aspirate was indicated to be associated 
with the development of BPD in premature infants (20). In addition, SUMOylation of SIRT1 increases its HDAC activity and serves as a molecular regulator that determines the fate of cells exposed to stress-induced DNA damage (5). Subsequently, it was hypothesized that SUMOylation of SIRT1 in premature neonates may be implicated in the development of BPD.

In the present study, the effects of hyperoxia on the expression of SUMO and SIRT1 proteins were examined, and the interactions of these proteins in premature neonates with BPD were investigated.

\section{Materials and methods}

Subjects and study design. In the present prospective study, 40 premature neonates (20 neonates with BPD and 20 gender-matched premature neonates without BPD; 22 male and 18 female; mean gestational age, 29.1 \pm 0.6 weeks; Table I) were enrolled at the Department of Neonatology in the Affiliated Hospital of Southwest Medical University (Luzhou, China) between July 12015 and August 31 2016. The following inclusion criteria were used: Gestational age between 28 and 30 weeks; treatment with non-invasive positive pressure ventilation or normal oxygen inhalation; and availability of blood samples for western blot analysis and immunoprecipitation assay. The following exclusion criteria applied to the present study: Presence of congenital heart diseases; multiple anomaly syndromes; chromosomal abnormalities; or hospitalization time $<28$ days. No other pre-existing conditions existed in the neonates included in the present study.

BPD was defined as the requirement of supplemental oxygen at the 28th postnatal day (4), and subjects were assigned to the BPD or non-BPD group (20 per group) based on this diagnostic criterion. According to fraction of inspired oxygen $\left(\mathrm{FiO}_{2}\right)$, obtained from ventilators, premature neonates were divided into low $\left(21 \%<\mathrm{FiO}_{2}<30 \%\right.$ lasting more than $24 \mathrm{~h})$, medium $\left(30 \% \leq \mathrm{FiO}_{2}<40 \%\right.$ lasting more than $24 \mathrm{~h}$ ), high-oxygen $\left(\mathrm{FiO}_{2} \geq 40 \%\right.$ lasting more than $\left.24 \mathrm{~h}\right)$, and normoxia $\left(\mathrm{FiO}_{2}>21 \%\right.$ lasting less than $24 \mathrm{~h}$ or $\left.\mathrm{FiO}_{2}=21 \%\right)$ groups (12). Residual venous blood samples at 28 days post hospitalization were obtained for isolation of peripheral blood mononuclear cells (PBMCs). The Ethics Committee at the Affiliated Hospital of Southwest Medical University approved the present study. Written informed consent was obtained from immediate family members of all premature neonates.

Reagents and antibodies. Human peripheral blood lymphocyte separation solution was purchased from Tianjin Haoyang Biological Manufacture Co., Ltd. (Tianjin, China). Radioimmunoprecipitation assay (RIPA) protein lysis buffer was obtained from Amyjet Scientific, Inc. (Wuhan, China). A BCA protein assay kit was purchased from Sigma-Aldrich; Merck KGaA (Darmstadt, Germany). Antibodies used included: Rabbit anti-human SIRT1 antibody (Santa Cruz Biotechnology Inc., Dallas, TX, USA), rabbit anti-human GAPDH antibody (Beyotime Institute of Biotechnology, Inc., Jiangsu, China), horseradish peroxidase (HRP)-labeled goat anti-rabbit immunoglobulin $\mathrm{G}$ ( $\mathrm{IgG}$ ) antibody (Shanghai YuanMu Biological Technology Co., Ltd., Shanghai, China), anti-SUMO1 monoclonal antibody (ab133352; Abcam, Cambridge, UK), anti-SUMO2/3 polyclonal antibody (ab3742;
Table I. Baseline characteristics of subjects.

\begin{tabular}{lcc}
\hline Variable & BPD group & Non-BPD group \\
\hline Number of neonates, $\mathrm{n}$ & 20 & 20 \\
Male/female, $\mathrm{n}$ & $11 / 9$ & $11 / 9$ \\
Gestational age, week & $28.9 \pm 0.5$ & $29.2 \pm 0.5$ \\
Body weight, $\mathrm{g}$ & $1,179.9 \pm 80.8$ & $1,213.8 \pm 76.8$ \\
Oxygen therapy & & \\
Normoxia group, $\mathrm{n}$ & 0 & 6 \\
Low-oxygen group, $\mathrm{n}$ & 0 & 12 \\
Medium-oxygen group, $\mathrm{n}$ & 16 & 0 \\
High-oxygen group, $\mathrm{n}$ & 4 & 2 \\
\hline
\end{tabular}

EMD Millipore, Billerica, MA, USA), and anti-SUMO4 monoclonal antibody (ab126606; Abcam).

Preparation of venous blood samples. Residual venous blood samples of premature neonates in the BPD and non-BDP groups were collected to isolate PBMCs by Ficoll density gradient centrifugation. Blood samples $(1.0-1.5 \mathrm{ml})$ were placed in an anticoagulant tube, diluted and mixed with an identical volume of normal saline, and then transferred to a centrifuge tube with lymphocyte separation solution at the bottom. Following centrifugation at $900 \mathrm{x} \mathrm{g}$ for $20 \mathrm{~min}$ at room temperature, the mononuclear cell layer was transferred to another centrifuge tube with a pipette and washed twice with normal saline. The supernatant was discarded and the remaining PBMC pellets were collected and stored at $-80^{\circ} \mathrm{C}$.

Protein extraction and western blot analysis. PBMCs were solubilized in RIPA protein lysis buffer for $30 \mathrm{~min}$ on ice followed by centrifugation at $900 \mathrm{x}$ g for $15 \mathrm{~min}$ at $4^{\circ} \mathrm{C}$. The supernatant was collected and protein concentrations were quantitated using the protein assay kit according to the manufacturer's protocols. Equivalent protein extracts $(1.8 \mu \mathrm{mol} / \mathrm{l})$ were separated by $10 \%$ SDS-PAGE and transferred electrophoretically onto polyvinylidene difluoride (pore size, $0.2 \mu \mathrm{m}$ ) membranes. Following incubation with blocking buffer containing 5\% non-fat dry milk for $1 \mathrm{~h}$ at room temperature, membranes were incubated overnight with rabbit anti-SIRT1 (ab110304; 1:1,000), anti-SUMO1 monoclonal (1:800), anti-SUMO2/3 polyclonal (1:800), anti-SUMO4 monoclonal $(1: 800)$ or rabbit anti-GAPDH antibodies $(1: 3,000)$ at $4^{\circ} \mathrm{C}$. The blots were washed three times for 5 min each with TBS/Tween-20 (TBST), and subsequently incubated with HRP-labeled goat anti-rabbit IgG antibody $(1: 3,000)$ for $1 \mathrm{~h}$ at room temperature. Following three washes (5 min each) with TBST, chemiluminescent signals were visualized using an enhanced chemiluminescence kit (Pierce; Thermo Fisher Scientific, Inc., Waltham, MA, USA) and band intensity was analyzed using Quantity One software (version 4.6.6; Bio-Rad Laboratories, Inc., Hercules, CA, USA). The expression levels of target proteins were normalized relative to that of GAPDH.

Immunoprecipitation assay. Protein extracts were prepared by adding the radioimmunoprecipitation lysis buffer (Pierce; 
A

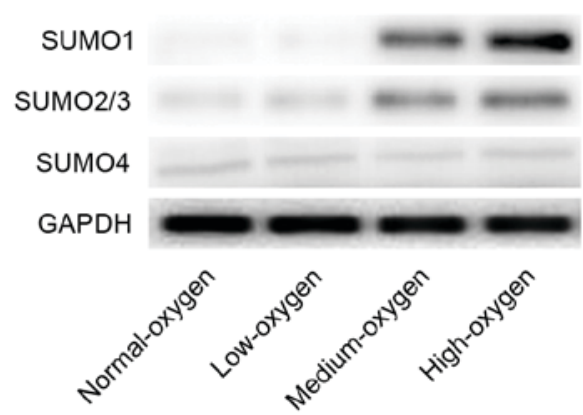

B

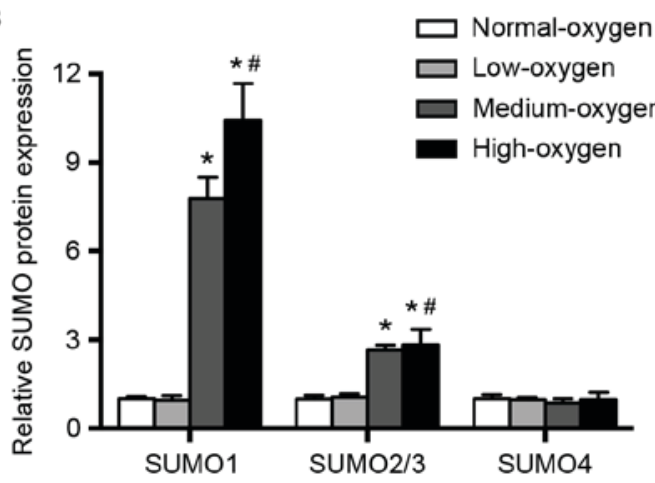

Figure 1. Western blot analysis of SUMO expression following inhalation of different concentrations of oxygen. Protein extracts lysed from PBMCs were immunoblotted with anti-SUMO1, anti-SUMO2/3, or anti-SUMO4 antibody. The relative expression levels of SUMO proteins were normalized to that of GAPDH. (A) Representative images; (B) quantitative data. SUMO1 and SUMO2/3 expression levels in the normoxia group were significantly lower compared with those in the medium- or high-oxygen group. Data were expressed as the mean + standard deviation ( $\mathrm{n}=3$ per group). " $\mathrm{P}<0.01$ vs. normoxia group; ${ }^{\mathrm{P}} \mathrm{P}<0.01$ vs. medium-oxygen group. $\mathrm{FiO}_{2}$, fraction of inspired oxygen; PBMCs, peripheral blood mononuclear cells; SUMO, small ubiquitin-related modifier.

Thermo Fisher Scientific, Inc.) containing protease inhibitors to PBMCs, and then incubated with rabbit anti-SIRT1 antibody (SC-135792) or control IgG antibody (Pierce, Rockford, IL, USA) overnight at $4^{\circ} \mathrm{C}$. Once antigen-antibody complexes were precipitated with protein A-sepharose beads (Pierce; Thermo Fisher Scientific, Inc.) for 30-60 min at room temperature, immunoprecipitated proteins $(1.8 \mu \mathrm{mol} / \mathrm{l})$ were separated by $10 \%$ SDS-PAGE and incubated overnight at $4{ }^{\circ} \mathrm{C}$ with anti-SUMO1 monoclonal (1:800), anti-SUMO2/3 polyclonal (1:600) or anti-SUMO4 monoclonal antibody (1:600). SIRT1 was used as the reference protein. Chemiluminescent signals were visualized using the enhanced chemiluminescence kit (Pierce; Thermo Fisher Scientific, Inc.) and band intensity was analyzed using Quantity One software (version 4.6.6; Bio-Rad Laboratories, Inc., Hercules, CA, USA).

Statistical analyses. All statistical analyses were performed using SPSS for Windows (version 17.0; SPSS, Inc., Chicago, IL, USA). Data pertaining to normally distributed variables were expressed as the mean \pm or + standard deviation as indicated, and between-group differences were assessed using Student's t-test. Multi-group comparisons were performed using one-way analysis of variance with Dunnett's post hoc test. Categorical data were compared using $\chi^{2}$ test. $\mathrm{P}<0.05$ was considered to indicate a statistically significant difference.

\section{Results}

Baseline characteristics. The present study included 40 premature neonates (22 male and 18 female; mean gestational age, $29.1 \pm 0.6$ weeks; Table 1). Of the 20 neonates in the BPD group, 16 neonates were indicated in the medium-oxygen group and 4 neonates were identified in the high-oxygen group. However, in the non-BPD group, 6 neonates were in the normoxia group, 12 in the low-oxygen group and 2 in the medium-oxygen group. No significant differences were observed between the BPD and non-BPD groups with regards to gestational age and body weight.

SUMO expression after inhalation of different concentrations of oxygen. Western blot analysis was performed to examine the expression levels of SUMO proteins in PBMCs

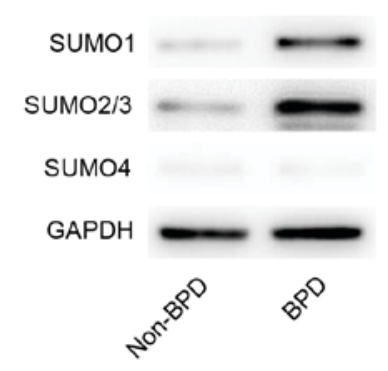

B

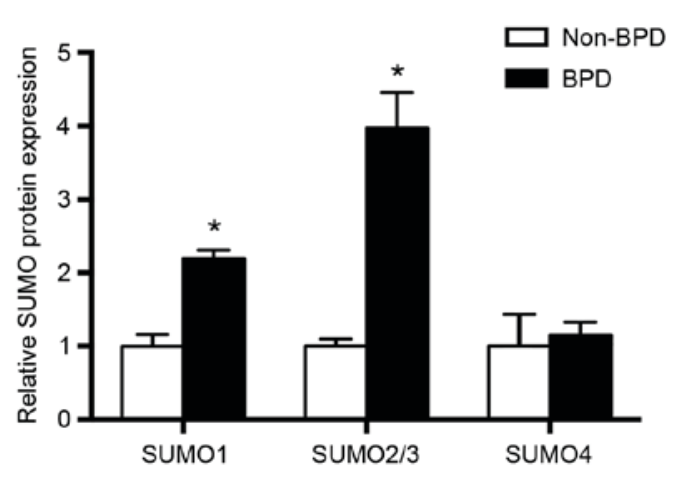

Figure 2. Western blot analysis of SUMO expression levels in the BPD and non-BPD groups. (A) Representative images; (B) quantitative data. Expression levels of SUMO1 and SUMO2/3 proteins in the BPD group were significantly higher compared with those in the non-BPD group. Data were expressed as the mean + standard deviation ( $n=3$ per group). ${ }^{*} \mathrm{P}<0.01$ vs. non-BPD group. BPD, bronchopulmonary dysplasia; SUMO, small ubiquitin-related modifier.

of premature infants. The expression levels of SUMO1 and SUMO2/3 proteins in the normoxia group were significantly lower than those in the medium- and high-oxygen groups $(\mathrm{P}<0.01)$, but were comparable to those in the low-oxygen group (Fig. 1). SUMO4 expression levels were comparable among the normoxia, low-, middle- and high-oxygen groups (Fig. 1). These results indicate that oxygen inhalation with $\mathrm{FiO}_{2} \geq 30 \%$ significantly upregulated SUMO1 and SUMO2/3 expression levels in premature infants. 
A

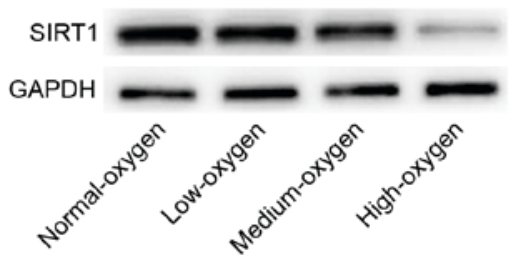

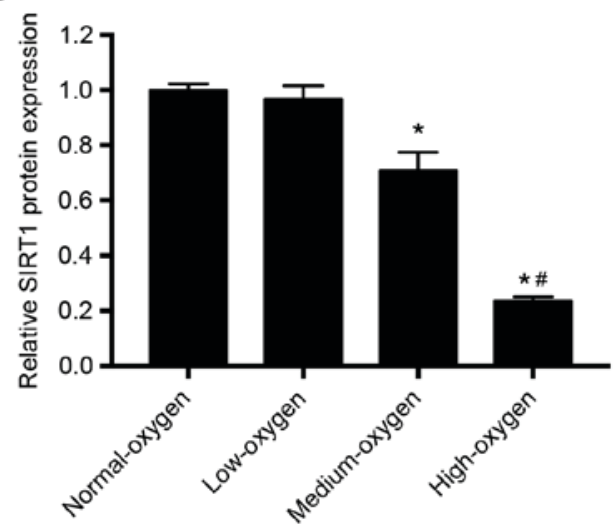

Figure 3. Western blot analysis of SIRT1 expression levels associated with supplemental oxygen therapy at different concentrations in preterm infants. Protein extracts lysed from peripheral blood mononuclear cells were immunoblotted with anti-SIRT1 antibody. (A) Representative images; (B) quantitative data. SIRT1 protein expression levels in the medium- and high-oxygen groups were significantly lower compared with those in the normoxia group. Data were expressed as the mean + standard deviation ( $\mathrm{n}=3$ per group). ${ }^{\mathrm{P}} \mathrm{P}<0.01$ vs. normoxia group; ${ }^{*} \mathrm{P}<0.01$ vs. medium-oxygen group. SIRT1, sirtuin 1 .

Comparison of SUMO expression in the BPD and non-BPD groups. The expression levels of SUMO1 and SUMO2/3 proteins in the BPD group were significantly higher compared with those in the non-BPD group $(\mathrm{P}<0.01)$, whereas no significant difference in SUMO4 expression was observed between groups (Fig. 2)

SIRT1 expression following inhalation of different concentrations of oxygen. The expression levels of SIRT1 protein in the medium- and high-oxygen groups were significantly lower compared with those in the normoxia group $(\mathrm{P}<0.01$; Fig. 3$)$. SIRT1 expression levels in the low-oxygen group were lower than that in the normoxia group; however, the difference was not statistically significant (Fig. 3). These results suggest that oxygen inhalation with $\mathrm{FiO}_{2} \geq 30 \%$ may significantly downregulate SIRT1 expression in preterm infants.

Comparison of SIRT1 expression in the BPD and non-BPD groups. SIRT1 expression was compared in the BPD and non-BPD groups to assess its potential role in BPD. As indicated in Fig. 4, the expression levels of SIRT1 protein in the BPD group was significantly lower than that in the non-BPD group $(\mathrm{P}<0.01)$.

Immunoprecipitation assay of interactions between SUMO and SIRT1. Interactions between SUMO and SIRT1 were detected by immunoprecipitation with anti-SIRT1 antibody and followed by western blot analysis using anti-SUMO1, anti-SUMO2/3 or anti-SUMO 4 antibodies. As indicated in Fig. 5, SIRT1 interacted with SUMO1 and SUMO2/3 in both the non-BPD and BPD groups. However, SIRT1-SUMO4 interaction was not observed in either the non-BPD or BPD group. Both SIRT1-SUMO1 and SIRT1-SUMO2/3 interactions in the BPD group were significantly weaker than those in the non-BPD group $(\mathrm{P}<0.01$; Fig. 5).

\section{Discussion}

In the present study, the expression levels of SUMO and SIRT1 in PBMCs of premature neonates who were

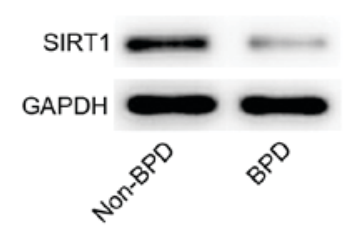

B

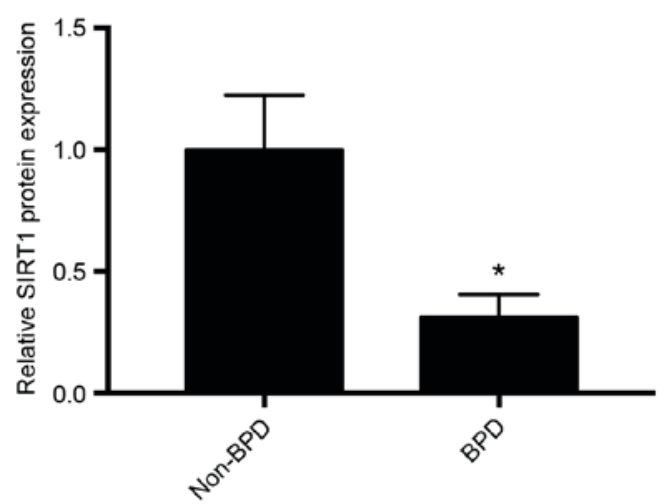

Figure 4. Western blot analysis of SIRT1 expression levels in the BPD and non-BPD groups. (A) Representative images; (B) quantitative data. Expression levels of SIRT1 protein in the BPD group were significantly lower than that in the non-BPD group. Data were expressed as the mean + standard deviation ( $\mathrm{n}=3$ per group). ${ }^{\mathrm{P}}<0.01$ vs. non-BPD group. $\mathrm{BPD}$, bronchopulmonary dysplasia; SIRT1, sirtuin 1.

administered different concentrations of oxygen were investigated. Furthermore, the expression levels of SIRT1 and SUMO proteins and their interactions in BPD and non-BPD groups were determined. The present findings demonstrated that oxygen inhalation with $\mathrm{FiO}_{2} \geq 30 \%$ significantly upregulated SUMO1 and SUMO2/3 expression, but downregulated SIRT1 expression. Compared with the non-BPD group, the expression of SIRT1 protein and SUMOylation of SIRT1 by SUMO1 and SUMO2/3 was significantly attenuated in the BPD group. To the best of our knowledge, SUMOylation of 
A

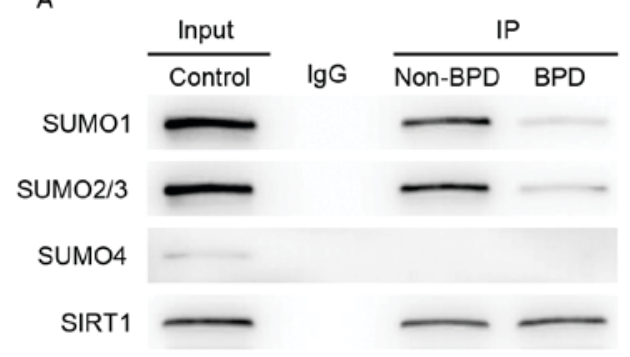

B

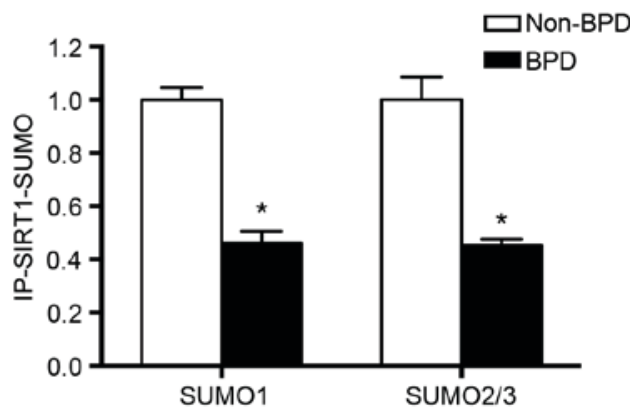

Figure 5. Immunoprecipitation assay indicated the interactions between SUMO and SIRT1. Protein extracts were immunoprecipitated with anti-SIRT1 or control IgG antibody and underwent western blot analysis with anti-SUMO1, anti-SUMO2/3 or anti-SUMO4 antibody. (A) Representative images; (B) quantitative data. SIRT1-SUMO1 and SIRT1-SUMO2/3 interactions in the BPD group were significantly attenuated compared with that in the non-BPD group. Data were expressed as the mean + standard deviation ( $\mathrm{n}=3$ per group). * $\mathrm{P}<0.01$ vs. non-BPD group. IP, immunoprecipitation; IgG, immunoglobulin G; SUMO, small ubiquitin-related modifier; BPD, bronchopulmonary dysplasia; SIRT1, sirtuin 1.

SIRT1 in premature neonates with BPD has not been previously investigated.

Supplemental oxygen is an important aspect of treatment of premature neonates with respiratory failure. However, hyperoxic exposure triggers oxidative stress, results in lung injury and contributes to the development of BPD $(3,4)$. Infants with BPD who were administered higher concentrations of supplemental oxygen were previously demonstrated to have more persistent lung disease (4). Increased oxidative stress has also been detected in adolescents with BPD (2). SUMO is crucial for the cellular response to oxidative stress (19). Huang et al (21) previously reported that exposure to high glucose upregulated SUMO1 and SUMO2/3 expression levels in a time- and dose-dependent. manner. In the present study, oxygen inhalation with $\mathrm{FiO}_{2} \geq 30 \%$ significantly increased SUMO1 and SUMO2/3 expression levels in PBMCs of preterm infants. However, no significant difference was observed between the normoxia, low-, middle- and high-oxygen groups with respect to SUMO4 expression. Although previous studies have indicated a role of SUMO4 in the modulation of cellular response to stress, its function is not well-characterized (16). In the present study, expression levels of SUMO1 and SUMO2/3 in the BPD group were significantly higher compared with those in the non-BPD group. These findings indicate that SUMO1 and SUMO2/3 may serve as oxidative stress-related proteins and may have a role in the pathogenesis of BPD in premature neonates. In a previous study by Yuan et al (22), individual deletion of either SUMO1 or SUMO2/3 did not affect the development of zebra fish, whereas inactivation of all three SUMOs triggered p53-dependent apoptosis and resulted in severe developmental defects.

HDAC is associated with the regulation of cell differentiation and proliferation. Decreased HDAC activity has been indicated to cause cell cycle arrest and suppress alveolar cell proliferation (23). SIRT1 is a NAD-dependent HDAC, which has a crucial role in the cellular response to nutritional and metabolic disorders, inflammation, hypoxia and oxidative stress (24-26). In a neonatal rat model of lung injury, exposure to hyperoxia was revealed to induce the downregulation of HDAC expression (27,28). Similar results were obtained in the present study, in that the expression levels of SIRT1 in the medium- and high-oxygen groups were significantly lower compared with those in the normoxia group. Furthermore, SIRT1 has been indicated to protect against oxidative stress and inhibit apoptosis $(7,8)$.
Abnormal expression of SIRT1 was recently observed in patients with pulmonary fibrosis, pulmonary inflammatory diseases or lung tumors (29-31). Mody et al (20) previously performed immunocytochemistry analysis to detect SIRT1 expression in tracheal aspirate leukocytes of 51 premature infants, and demonstrated SIRT1 to be less localized in the nuclei of tracheal aspirate mononuclear cells in infants with BPD compared with those who did not develop BPD. In the present study, SIRT1 expression in PBMCs of preterm infants in the BPD group was significantly lower than that in the non-BPD group. Additionally, SIRT1 has been suggested to promote the survival of both aging and cancerous cells (32). Lower SIRT1 in tracheal aspirate leukocytes has previously been indicated to be associated with the development of BPD in premature infants (20). In addition, SIRT1 is considered as a potential therapeutic target in the context of diseases including cancer, Alzheimer's diseases and diabetes (7).

SUMOylation is a reversible posttranslational modification that contributes to various cellular processes, including genome stability, gene expression, RNA processing, protein synthesis and repair of DNA damage, and has a crucial role in a variety of human diseases, including brain ischemia, heart diseases, cancer and degenerative diseases $(14,15)$. SUMOylation of target proteins leads to alterations of their subcellular localization, activity and stability (17). Furthermore, SUMOylation of SIRT1 by SUMO1 was identified in DU145 prostate cancer cells, and HDAC activity of SIRT1 was enhanced after SUMOylation (5). A recent study by Han et al (32) indicated that SUMOylation of SIRT1 improved the stability of SIRT1 protein. In the present study, SUMOylation of SIRT1 by SUMO1 and SUMO2/3 was demonstrated in PBMCs of premature neonates; however, no notable interaction was observed between SUMO4 and SIRT1. A previous study has also indicated that the possible role of SUMO4 in SUMOylation is yet to be understood (17). In a previous study by Yang et al (5), decreased SUMOylation of SIRT1 in response to acute DNA damage attenuated its HDAC activity, enhanced the activity of its pro-apoptotic substrates and ultimately resulted in cell death. In the present study, SUMOylation of SIRT1 by SUMO1 and SUMO2/3 in the BPD group was significantly attenuated when compared with that in the non-BPD group. These findings suggest that decreased SUMOylation of SIRT1 may be associated with the pathogenesis of BPD in premature neonates. 
This prospective study has limitations. Firstly, the expression of SIRT1 and SUMO proteins and their interactions were examined in PBMCs rather than in lung biopsy tissues. However, PBMCs are widely distributed in the lung tissue, and likely have a role in the development of BPD. Secondly, only a small sample of premature neonates was included in the present study.

In conclusion, oxygen inhalation with $\mathrm{FiO}_{2} \geq 30 \%$ was significantly associated with the downregulation of SIRT1 expression in PBMCs of premature neonates. Decreased expression of SIRT1, and its interactions with SUMO1 and SUMO2/3 may be associated with the development of BPD. As the present study is only a preliminary work, further investigation with a larger sample size is required to elucidate the mechanism of SIRT1-associated SUMOylation in the development of BPD.

\section{Acknowledgements}

The present study was supported by grants from projects in the National Natural Science Foundation of China (grant no. 81571480) and the Joint Special Fund of Luzhou Municipal Government and Luzhou Medical College (grant no. 2013LZLY-J08).

\section{References}

1. Bhandari A and Bhandari V: Pitfalls, problems, and progress in bronchopulmonary dysplasia. Pediatrics 123: 1562-1573, 2009.

2. Niedermaier $\mathrm{S}$ and Hilgendorff A: Bronchopulmonary dysplasia-an overview about pathophysiologic concepts. Mol Cell Pediatr 2: 2, 2015.

3. Li Q, Wall SB, Ren C, Velten M, Hill CL, Locy ML, Rogers LK and Tipple TE: Thioredoxin reductase inhibition attenuates neonatal hyperoxic lung injury and enhances nuclear factor E2-Related Factor 2 activation. Am J Respir Cell Mol Biol 55: 419-428, 2016.

4. Ali Z, Schmidt P, Dodd J and Jeppesen DL: Bronchopulmonary dysplasia: A review. Arch Gynecol Obstet 288: 325-333, 2013.

5. Yang Y, Fu W, Chen J, Olashaw N, Zhang X, Nicosia SV, Bhalla K and Bai W: SIRT1 sumoylation regulates its deacetylase activity and cellular response to genotoxic stress. Nat Cell Biol 9: 1253-1262, 2007.

6. Chang HC and Guarente L: SIRT1 mediates central circadian control in the SCN by a mechanism that decays with aging. Cell 153: 1448-1460, 2013.

7. Ou X, Lee MR, Huang X, Messina-Graham S and Broxmeyer HE: SIRT1 positively regulates autophagy and mitochondria function in embryonic stem cells under oxidative stress. Stem Cells 32: 1183-1194, 2014.

8. Ruan Y, Dong C, Patel J, Duan C, Wang X, Wu X, Cao Y, Pu L, $\mathrm{Lu}$ D, Shen T and Li J: SIRT1 suppresses doxorubicin-induced cardiotoxicity by regulating the oxidative stress and p38MAPK pathways. Cell Physiol Biochem 35: 1116-1124, 2015.

9. Hori YS, Kuno A, Hosoda R and Horio Y: Regulation of FOXOs and $\mathrm{p} 53$ by SIRT1 modulators under oxidative stress. PLoS One 8: e73875, 2013.

10. Li Y, Wang P, Yang X, Wang W, Zhang J, He Y, Zhang W, Jing T, Wang B and Lin R: SIRT1 inhibits inflammatory response partly through regulation of NLRP3 inflammasome in vascular endothelial cells. Mol Immunol 77: 148-156, 2016.

11. Kong X, Guan J, Li J, Wei J and Wang R: P66Shc-SIRT1 regulation of oxidative stress protects against cardio-cerebral vascular disease. Mol Neurobiol 54: 5277-5285, 2017.

12. Zhang C, Li Q, Kang L, Lei X, Zhai X, Zhao S, Zhang C and Dong W: Resveratrol inhibits hyperxia-induced cell apoptosis through up-regulating SIRT1 expression in HPAECs. Xi Bao Yu Fen Zi Mian Yi Xue Za Zhi 31: 590-595, 2015 (In Chinese).
13. Yang X, Dong W, Li Q, Kang L, Lei X, Zhang L, Lu Y and Zhai X: Hyperoxia induces reactive oxygen species production and promotes SIRT1 nucleocytoplasmic shuttling of peripheral blood mononuclear cells in premature infants in vitro. Xi Bao Yu Fen Zi Mian Yi Xue Za Zhi 31: 1669-1676, 2015 (In Chinese).

14. Feligioni M and Nistico R: SUMO: A (oxidative) stressed protein. Neuromolecular Med 15: 707-719, 2013.

15. Yang W and Paschen W: SUMO proteomics to decipher the SUMO-modified proteome regulated by various diseases. Proteomics 15: 1181-1191, 2015.

16. Chymkowitch P, Nguéa PA and Enserink JM: SUMO-regulated transcription: Challenging the dogma. Bioessays 37: 1095-1105, 2015.

17. Sriramachandran AM and Dohmen RJ: SUMO-targeted ubiquitin ligases. Biochim Biophys Acta 1843: 75-85, 2014.

18. Agbor TA and Taylor CT: SUMO, hypoxia and the regulation of metabolism. Biochem Soc Trans 36: 445-448, 2008.

19. Enserink JM: Sumo and the cellular stress response. Cell Div 10: 4, 2015.

20. Mody K, Saslow JG, Kathiravan S, Eydelman R, Bhat V, Stahl GE, Pyon K, Bhandari V and Aghai ZH: Sirtuin1 in tracheal aspirate leukocytes: Possible role in the development of bronchopulmonary dysplasia in premature infants. J Matern Fetal Neonatal Med 25: 1483-1487, 2012.

21. Huang W, Xu L, Zhou X, Gao C, Yang M, Chen G, Zhu J, Jiang L, Gan H, Gou F, et al: High glucose induces activation of NF- $\kappa \mathrm{B}$ inflammatory signaling through $\mathrm{I} \kappa \mathrm{B} \alpha$ sumoylation in rat mesangial cells. Biochem Biophys Res Commun 438: 568-574, 2013.

22. Yuan H, Zhou J, Deng M, Liu X, Le Bras M, de The H, Chen SJ, Chen Z, Liu TX and Zhu J: Small ubiquitin-related modifier paralogs are indispensable but functionally redundant during early development of zebrafish. Cell Res 20: 185-196, 2010.

23. Huang YX, Zhao J, Song QH, Zheng LH, Fan C, Liu TT, Bao YL, Sun LG, Zhang LB and Li YX: Virtual screening and experimental validation of novel histone deacetylase inhibitors. BMC Pharmacol Toxicol 17: 32, 2016.

24. Li Y, Ni J, Guo R and Li W: In patients with coronary artery disease and type 2 diabetes, SIRT1 expression in circulating mononuclear cells is associated with levels of inflammatory cytokines but not with coronary lesions. Biomed Res Int 2016: 8734827, 2016.

25. Zhu Y, Sun Y, Guan W, Yan Y, Zhang W, Bai L, Kong H and Li F: Lycium barbarum polysaccharides enhances SIRT1 expression and decreases MMP-9 and HIF- $1 \alpha$ expressions in hypoxic pulmonary vascular smooth muscle cells. Xi Bao Yu Fen Zi Mian Yi Xue Za Zhi 32: 906-910, 2016 (In Chinese).

26. Xue F, Huang JW, Ding PY, Zang HG, Kou ZJ, Li T, Fan J, Peng ZW and Yan WJ: Nrf2/antioxidant defense pathway is involved in the neuroprotective effects of Sirtl against focal cerebral ischemia in rats after hyperbaric oxygen preconditioning. Behav Brain Res 309: 1-8, 2016.

27. Cetinkaya M, Cansev M, Cekmez F, Tayman C, Canpolat FE, Kafa IM, Yaylagul EO, Kramer BW and Sarici SU: Protective effects of valproic acid, a histone deacetylase inhibitor, against hyperoxic lung injury in a neonatal rat model. PLoS One 10: e0126028, 2015.

28. Korfei M, Skwarna S, Henneke I, MacKenzie B, Klymenko O, Saito S, Ruppert C, von der Beck D, Mahavadi P,Klepetko W, et al: Aberrant expression and activity of histone deacetylases in sporadic idiopathic pulmonary fibrosis. Thorax 70: 1022-1032, 2015.

29. Conti V, Corbi G, Manzo V, Pelaia G, Filippelli A and Vatrella A: Sirtuin 1 and aging theory for chronic obstructive pulmonary disease. Anal Cell Pathol (Amst) 2015: 897327, 2015.

30. Ahmad T, Sundar IK, Tormos AM, Lerner CA, Gerloff J, Yao H and Rahman I: Shelterin telomere protection protein 1 reduction causes telomere attrition and cellular senescence via sirtuin 1 deacetylase in chronic obstructive pulmonary disease. Am J Respir Cell Mol Biol 56: 38-49, 2017.

31. Liu HY, Li QR, Cheng XF, Wang GJ and Hao HP: NAMPT inhibition synergizes with NQO1-targeting agents in inducing apoptotic cell death in non-small cell lung cancer cells. Chin J Nat Med 14: 582-589, 2016.

32. Han X, Niu J, Zhao Y, Kong Q, Tong T and Han L: HDAC4 stabilizes SIRT1 via sumoylation SIRT1 to delay cellular senescence. Clin Exp Pharmacol Physiol 43: 41-46, 2016. 